Contents List available at VOLKSON PRESS

Intelligent Computing and Information Engineering (ICIE )

DOI : http://doi.org/10.26480/icie.01.2017.33.35

Journal Homepage: : https://www.intelcomp-design.com/

\title{
Study on Load of Middle and Small Span Highway Beam Bridge
}

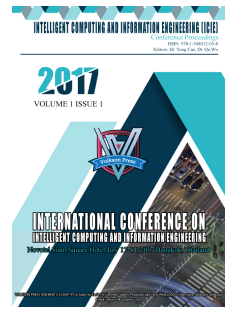

Ding Wenxia

Hubei Communication Technical College, Wuhan

*email25716717@qq.com

This is an open access article distributed under the Creative Commons Attribution License, which permits unrestricted use, distribution, and reproduction in any medium, provided the original work is properly cited

\section{ARTICLE DETAILS}

\section{Article History:}

Received 12 May2017

Accepted 12 July 2017

Available online 14 September 2017

Keywords:

Load, Dynamic characteristics,

Highway Bridge, ANSYS..

\section{ABSTRACT}

The magnitude of the load is the main reason for the direct impact of highway bridge safety. With the increase in transport demand, vehicle overload phenomenon gradually increased. In recent years, repeated heavy vehicles crushed the bridge accident, causing widespread concern in society. In this paper, the load characteristics of the bridge are analyzed by ANSYSY finite element simulation. And provide a useful reference for the design and construction of highway bridge structure.

\section{Introduction}

The vehicle is on the road and has an effect on the structure of the road. This is a complicated process, and its internal forces are more complicated. Because of the spatial integrity of the structure, when the bridge on the role of live load, the main beam will participate in the work, so that the formation of the main beam between the internal force distribution; the other hand, the internal force distribution with the live load Changes in position. In view of the vehicle load on the road surface can be moved along the vertical and horizontal can be a number of local loads, with the influence of the surface to solve the most adverse internal force of the mathematical formula complex, the calculation is very cumbersome, usually non-Gaussian random process simulation method, Complex spatial problems are transformed into simple plane problems.

The load experiment of highway bridge structure is a kind of method to judge the bearing capacity of Highway Bridge and evaluate its service life more common and intuitive [1]. Vehicle load fatigue test through the load caused by the structure of the displacement and fracture, to test the structure of the specified parts of the stress, strain, stiffness, deflection and other data. There are a lot of literature on the concrete structure of the fatigue test mainly to equal amplitude (constant amplitude, constant amplitude) fatigue experiments, a small amount of variable fatigue test is gene rally only two or three variable amplitude fatigue experiments. While the actual bridge structure is subjected to a load that is constantly changing the random load [2]. Although the fatigue test is relatively simple, but with the actual operation of the load spectrum is very different, and the experimental process can not consider the interaction between different load cycles, so the same scale fatigue test cannot really reflect the actual work of highway bridge operations Status and force status.

\section{MODEL ANALYSIS}

\subsection{Bridge Model}

Bridge longitudinal span of 7.8 meters, horizontal span of 6 meters, the structure shown in Figure 1 below.

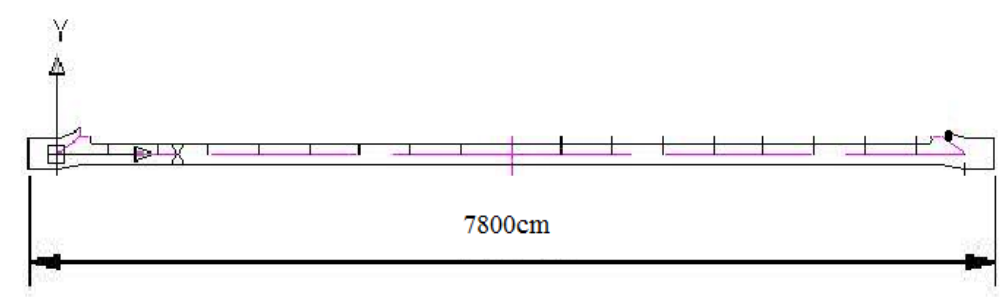

Figure 1: Structure Diagram

\subsection{Finite Element Model Establishment}

In the establishment of the finite element model, we use the three-dimensional shell element, and it's under the font steel beam is a three-dimensional beam element, and the beam element is as a shell element of the ribs to be considered [3]. The grid of the concrete panel is divided into $0.5 \times 0.6 \mathrm{~m}$ grid on both sides of the cantilever, and the grid of the middle concrete panel is $0.55 \times 0.6 \mathrm{~m}$, so there are 1700 shell elements in the concrete panel. I-shaped steel beam in its longitudinal use of the 0.6 -meter grid, 8 beams a total of 400 three-dimensional beam element. The preliminary analysis shows that such a grid density can meet the convergence requirements of the calculation results.

\subsection{Model Parameter Value Setting}


In the parameter settings, we select six basic random variables. They are three material strength variables and three structural geometric variables. The three material strength variables are the compressive strength of the concrete cube, the yield strength of the steel beam and the yield strength of the steel in the bridge panel [4]. The geometrical variables of the three structures are: the thickness of the concrete panel, the height of the steel beam and the diameter of the steel bar in the Table $l$.

Table 1: Material strength setting

\begin{tabular}{|c|c|c|c|}
\hline Variable & Distributed & Rated Value & $\begin{array}{c}\text { The Ratio of the Mean to } \\
\text { the Rated Value }\end{array}$ \\
\hline Concrete cube compressive strength & Logarithmic normal & $27 \mathrm{MP}$ & 1.386 \\
\hline Steel beam yield strength & Logarithmic normal & $241 \mathrm{MP}$ & 1.123 \\
\hline Steel yield strength & Logarithmic normal & $269 \mathrm{MP}$ & 1.078 \\
\hline Panel thickness & Normal distribution & $261 \mathrm{~mm}$ & 1.0 \\
\hline Steel beam height & Normal distribution & $1180 \mathrm{~mm}$ & 1.0 \\
\hline Reinforcement diameter & Normal distribution & $23 \mathrm{~mm}$ & 1.0 \\
\hline
\end{tabular}

The material properties in the finite element analysis are shown in Table 2:

Table 2: Material properties

\begin{tabular}{|c|c|c|c|c|}
\hline Position & Material & Elastic Modulus & Poisson's Ratio & $\begin{array}{c}\text { Mass Density } / \mathrm{Kg} \cdot \\
\mathrm{m}^{-3}\end{array}$ \\
\hline Beam & Prefabricated concrete C50 & $3.45 \times 10^{10}$ & 0.2 & 2600 \\
\hline Bridge Deck & Prefabricated concrete C40 & $1.95 \times 10^{11}$ & 0.2 & 7850 \\
\hline
\end{tabular}

\section{NUMERICAL SIMULATION}

Using the numerical simulation method to determine the traffic flow. Numerical simulation is the use of computers randomly generated with the observed data has the same statistical characteristics of the random number sequence, in order to simulate the objective random process [5]. And verifies and evaluates the reliability of the simulation results by testing the numerical characteristics of the generated random sequence. The key to the random sequence simulation is to design a distributed distribution of the random variables from the specified distribution function and construct the appropriate power distribution function of the sequence according to the actual background interference. The non - Gaussian stochastic process simulation method is used to simulate and compile the vehicle load spectrum of the Weibull distribution.

Determine the objective function:

$$
s(f)=\frac{1}{2}\left[\frac{1}{a^{2}+4 \pi^{2}\left(f+f_{0}\right)^{2}}+\frac{1}{a^{2}+4 \pi^{2}\left(f-f_{0}\right)^{2}}\right]
$$

The parameters $a$ and $f_{0}$ take the power density general function.

Set the mean and variance

The Gaussian process with Gaussian process is zero mean, and its variance is equal to the sample variance calculated from the fitted Weibull parameter. which is:

$\sigma_{G}^{2}=\sigma^{2}, \mu_{G}=0$

In general, the variationally principle is used to establish the generalized function on the basis of the numerical calculation method of elastic dynamics, and the governing equations of elastic dynamics and the conditions of solving are obtained by their standing conditions.

Usually we set the initial condition to $u(x, o)=u_{0}(x), u\left(x, t_{1}\right)=u_{1}\left(x_{1}\right)$, where $u_{0}(x)$ and $u_{1}(x)$ are given functions [6]. We study the above-mentioned geometrical equations and the established physical equations and through their displacement boundary conditions to calculate the possible state of motion to satisfy the above equation. Our establishment is carried out by Hamilton's variational principle. To meet the equations of the standing conditions established, the variational principle we stated as follows:

$\delta \int_{0}^{1} L d t=\delta \int_{0}^{1}(K-U) d t=0$

$\int_{0}^{1}\left[\int_{v}\left(-\rho u_{i}+\sigma_{i j, j}+\rho f_{i}\right) \delta u_{i} d V+\int_{S \sigma}\left(-p_{i}+p_{i}\right) \delta u_{i} d S\right] d t=0$

With the divergence theorem:

$\delta \prod=\int_{0}^{1}\left[\int_{v}\left(\rho u_{i}+\sigma_{i j, j}-\rho f_{i}\right) \delta u_{i} d V-\int_{S \sigma} p_{i} \delta u_{i} d S\right] d t=0$

The second order ordinary differential equation of structural dynamic response obtained by finite element discretization.

\section{CHARACTERISTIC ANALYSIS}

In the ANSYS software, the finite element model of bridge structure is established, and the solid element and other elements are combined to establish the SOLID65 unit simulation beam. As shown in Figure 3. 


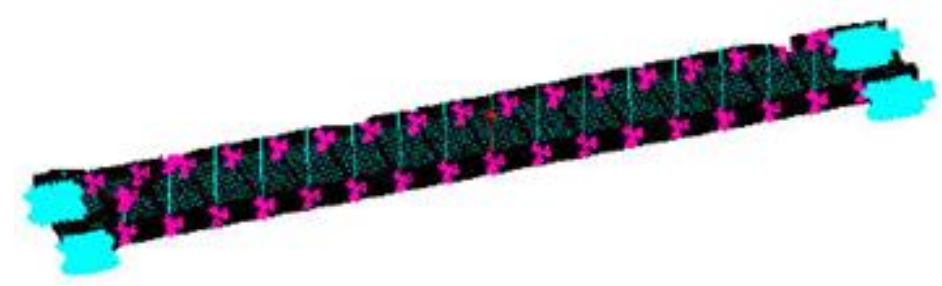

Figure 3: Finite element structure

Speed response curve with time, the design speed of $35 \mathrm{~m} / \mathrm{s}$ speed through the bridge when the dynamic response. The response curve shown in Figure 4 .

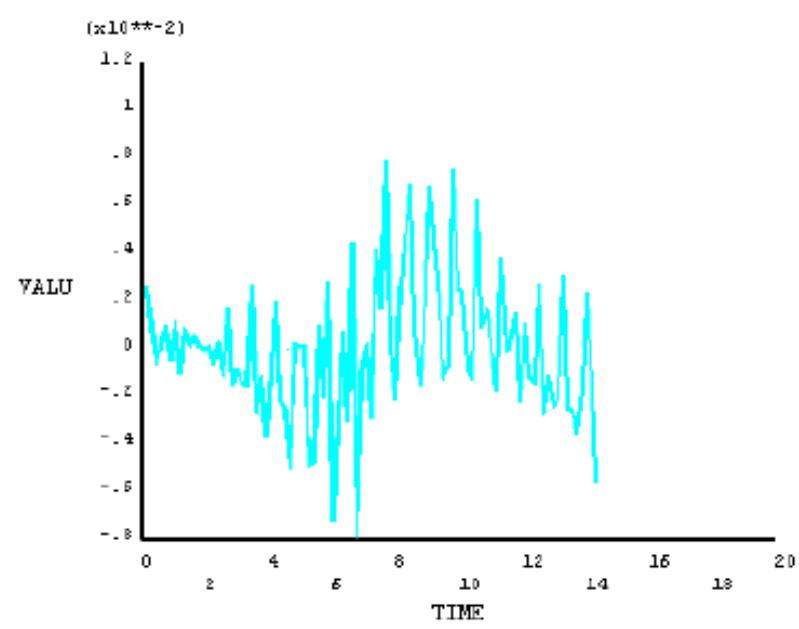

Figure 4: Response curve

From the figure can be obtained at a speed of $35 \mathrm{~km} / \mathrm{h}$ when the bridge in the maximum deflection of the bridge appears in $6.8435 \mathrm{~s}$, then the maximum deflection of $8.642 \mathrm{~cm}$ Department. At this time the maximum negative velocity is $0.005387 \mathrm{~m} / \mathrm{s}$, the maximum forward velocity appears at $7.7246 \mathrm{~s}$, the maximum forward speed is $0.007395 \mathrm{~m} / \mathrm{s}$; the maximum value of forward acceleration appears at $8.7406 \mathrm{~s}$, this When the maximum forward acceleration is $0.022096 \mathrm{~m} / \mathrm{s}^{2}$, the maximum value of the reverse acceleration appears at $14.4012 \mathrm{~s}$, and the maximum value of the reverse acceleration is $0.031899 \mathrm{~m}$ $/ s^{2}$.

\section{CONCLUSION}

The load environment of Highway Bridge has always been an important factor in bridge durability research and state assessment, and it is also a common concern in today's engineering industry. In this paper, the dynamic characteristics of the load of medium and small span highway girder bridge are studied by ANSYS, which is based on the basic theory knowledge of bridge dynamics and large-scale finite element analysis software ANSYS. Through the establishment of the finite element model of the whole solid element and the beam element space, the transient analysis simulates the dynamic response curve of the process when the traffic speed is $35 \mathrm{~km} / \mathrm{h}$, and provides a useful reference for the design and construction of the highway bridge structure.

\section{REFERENCES}

[1] Hong, Z.X., Yan, H.P., Wen, Y.G., Chen, Z., Tian, H.L. 2005. Statistics analysis and simulation of random loads for bridges. Journal of Jinan University (Natural Science and Medicine Edition), 26 (1), 114-116.

[2] Mei, L., Shengxie, X. 2000. Dynamic analysis of Beam Bridge under vehicle load. Journal of Chongqing Jiaotong University, 01.

[3] Yifan, S. 2000. Highway Bridge Dynamics M: Beijing: People's Communications Press, 6.

[4] Hua, L., Shu, Y.S., Tao, Z. 2006. Study on Dynamic Response of Continuous Beam under Driving Vehicle. Journal of Traffic and Transportation Engineering, 02.

[5] Peng, Z.J. 2006. Study on Some Problems of Bridge Structure Safety Design. Security Technology, 5, 37-38.

[6] Yong, S., Xian, C.C. 2005. Application of ANSYS in Bridge Engineering. Journal of Northeast Forestry University, 33 (2), 104-105. 